\title{
STRONGLY PURE SUBGROUPS OF SEPARABLE TORSION-FREE ABELIAN GROUPS
}

BY

\author{
LOYISO G. NONGXA \\ In Memory of my daughter Mosa Limakatso Nongxa
}

\begin{abstract}
In this paper we prove that countable strongly pure subgroups of completely decomposable groups are completely decomposable. We also show that strongly pure subgroups of separable torsion-free groups are separable.
\end{abstract}

One of the questions that have been considered concerning completely decomposable groups is the following: under what conditions is a subgroup of a completely decomposable group again completely decomposable? The well-known BaerKaplansky-Kulikov theorem asserts that direct summands of completely decomposable groups are also completely decomposable. It is well known that pure subgroups of completely decomposable groups are not necessarily completely decomposable and, in 1972, L. Bican characterized all those completely decomposable abelian groups any pure subgroup of which is completely decomposable. Recently, we have shown that homogeneous pure subgroups of a completely decomposable group $G$ are completely decomposable provided that the extractible typeset of $G$ is countable.

In this note we partially extend the Baer-Kaplansky-Kulikov theorem to strongly pure subgroups of completely decomposable groups. Specifically, we show that in a completely decomposable group whose typeset satisfies the maximum condition all strongly pure subgroups are completely decomposable. We also show that countable strongly pure subgroups of completely decomposable groups are completely decomposable. This not only extends but also gives a new proof of the classical theorem of Kulikov on countable direct summands of completely decomposable groups. Finally, we shall show that strongly pure subgroups of separable torsion-free groups are separable, extending the well-known theorem of L. Fuchs on direct summands of separable groups.

Throughout this note the word "group" will mean an "abelian group" and for notation and terminology the standard reference is volume II of Fuchs's book [2]. Let $G$ be torsion-free and completely decomposable, i.e. $G$ is a direct sum of rank one torsion-free groups. A type $\tau$ is said to be an extractible type of $G$ if $G$ has a rank one summand of type $\tau$. The extractible typeset of $G$, denoted by $\mathcal{E}(G)$, is the set of all extractible types of $G$. For every $\tau \in \mathcal{E}(G)$, we have $G(\tau)=G_{\tau} \oplus G^{*}(\tau)$, where $G_{\tau}$ is a nonzero homogeneous completely decomposable group of type $\tau$; $G_{\tau}$ is called a maximal $\tau$-homogeneous summand of $G$. If $G=\bigoplus_{\tau \in \mathcal{E}(G)} G_{\tau}$ is a decomposition of $G$ such that $G_{\tau}$ is a maximal $\tau$-homogeneous summand for every $\tau \in \mathcal{E}(G)$, then this decomposition is known as a homogeneous decomposition of $G$.

Received by the editors February 3, 1984.

1980 Mathematics Subject Classification. Primary 20K20.

(C) 1985 American Mathematical Society $0002-9947 / 85 \$ 1.00+\$ .25$ per page 
Let $G$ be a group and $H$ a subgroup of $G$. Then $H$ is said to be strongly pure in $G$ if, for every $h \in H$, there exists a homomorphism $\Psi: G \rightarrow H$ such that $\Psi(h)=h$. Strongly pure subgroups of arbitrary groups have been studied by S. Janakiraman and K. M. Rangaswamy [4]. They showed that the definition above implies that, if $H$ is strongly pure in $G$ and $\left\{h_{1}, h_{2}, \ldots, h_{n}\right\}$ is any finite subset of $H$, then there exists a homomorphism $\Psi: G \rightarrow H$ such that $\Psi\left(h_{i}\right)=h_{i}, i=1,2, \ldots, n$. This implies that a finitely generated strongly pure subgroup is a direct summand and finite rank strongly pure subgroups of torsion-free groups are also direct summands. It is also easy to see that strong purity is transitive and is an inductive property. Strongly pure subgroups are pure but purity does not necessarily imply strong purity for, if $G$ is a finite rank indecomposable torsion-free group with rank $G \geq 2$, then $G$ contains nontrivial pure subgroups whereas the only strongly pure subgroups of $G$ are $\{0\}$ and $G$ itself.

Let $G$ be a completely decomposable group and $\left\{\tau_{1}, \tau_{2}, \ldots, \tau_{n}\right\}$ a finite subset of $\mathcal{E}(G)$. If $G_{\tau_{i}}$ is a maximal $\tau_{i}$-homogeneous summand of $G$ for every $i=1,2, \ldots, n$, it is easy to see that $\sum_{i=1}^{n} G_{\tau_{i}}=\bigoplus_{i=1}^{n} G_{\tau_{i}}$ is a direct summand of $G$. This observation will be used repeatedly throughout this note.

One of the theorems proved by Baer is that, if $H$ is a direct summand of a completely decomposable group $G$ and the typeset of $H$ satisfies the maximum condition, then $H$ is itself completely decomposable. We have the following generalization of this theorem. (Also see [1, Proposition 10].)

THEOREM 1. Let $G$ be a completely decomposable group and $H$ a strongly pure subgroup of $G$. If the typeset, $T(H)$, of $H$ satisfies the maximum condition, then $H$ is completely decomposable.

PROOF. Let $G=\bigoplus_{\tau \in \mathcal{E}(G)} G_{\tau}$ be a homogeneous decomposition of $G$ and, for every $\tau \in \mathcal{E}(G)$, let $\pi_{\tau}: G \rightarrow G_{\tau}$ be the projection such that $\operatorname{ker} \pi_{\tau}=\bigoplus_{\tau^{\prime} \neq \tau} G_{\tau^{\prime}}$. Define

$$
\mathcal{E}(H)=\left\{\tau \in T(H):\left\langle H^{*}(\tau)\right\rangle_{*} \varsubsetneqq H(\tau)\right\} .
$$

If $\tau$ is a maximal type in $T(H)$ (which exists by assumption on $T(H)$ ), then trivially $\tau \in \mathcal{E}(H)$ and therefore $\mathcal{E}(H)$ is nonempty. Let $\tau_{0}$ be a fixed but arbitrary type in $\mathcal{E}(H)$ and let $0 \neq h \in H\left(\tau_{0}\right)-\left\langle H^{*}\left(\tau_{0}\right)\right\rangle_{*}$. If we define

$$
\mathcal{E}(h)=\left\{\tau \in \mathcal{E}(G): \pi_{\tau}(h) \neq 0\right\},
$$

then $h=\sum_{\tau \in \mathcal{E}(h)} \pi_{\tau}(h)$ and $\tau \geq \tau_{0}$ for every $\tau \in \mathcal{E}(h)$. By definition there exists a homomorphism $\Psi: G \rightarrow H$ such that

$$
h=\Psi(h)=\sum_{\tau \in \mathcal{E}(h)} \Psi \pi_{\tau}(h) .
$$

Assume that $\tau_{0} \notin \mathcal{E}(h)(\subseteq \mathcal{E}(G))$. Then, for every $\tau \in \mathcal{E}(h)$,

$$
\tau_{0} \supsetneqq \tau=\operatorname{type}_{G}\left(\pi_{\tau}(h)\right) \leq \operatorname{type}_{H}\left(\Psi \pi_{\tau}(h)\right) .
$$

This implies that $h \in\left\langle H^{*}\left(\tau_{0}\right)\right\rangle_{*}$; a contradiction. Thus $\tau_{0} \in \mathcal{E}(G)$ and $\pi_{\tau_{0}}(h) \neq 0$. We also conclude that $\mathcal{E}(H) \subseteq \mathcal{E}(G)$. The argument above shows that $\left\langle H^{*}(\tau)\right\rangle_{*}=$ 
$H \cap G^{*}(\tau)$ for every $\tau \in T(H)$. We then have for every $\tau \in \mathcal{E}(H)$,

$$
\begin{aligned}
H(\tau) /\left\langle H^{*}(\tau)\right\rangle_{*} & =(H \cap G(\tau)) /\left(H \cap G^{*}(\tau)\right) \\
& =(H \cap G(\tau)) /\left(H \cap G(\tau) \cap G^{*}(\tau)\right) \\
& \cong\left\langle H \cap G(\tau), G^{*}(\tau)\right\rangle / G^{*}(\tau) \\
& \leq G(\tau) / G^{*}(\tau) \cong G_{\tau},
\end{aligned}
$$

a homogeneous completely decomposable group of type $\tau$. Thus $H(\tau) /\left\langle H^{*}(\tau)\right\rangle_{*}$ is a homogeneous group of type $\tau$ and, by Lemma 86.4 in [2], $\left\langle H^{*}(\tau)\right\rangle_{*}$ is a balanced subgroup of $H(\tau)$. By Theorem 86.6 in $[2], H(\tau) /\left\langle H^{*}(\tau)\right\rangle_{*}$ is a completely decomposable group and, since completely decomposable groups are balance projective, $H(\tau)=H_{\tau} \oplus\left\langle H^{*}(\tau)\right\rangle_{*}$, where $H_{\tau}$ is a nonzero homogeneous completely decomposable group of type $\tau$. Since $H(\tau)$ is strongly pure in $G, H_{\tau}$ is also strongly pure in $G$ and therefore every finite rank pure subgroup of $H_{\tau}$ is a summand of $G$ for every $\tau \in \mathcal{E}(H)$.

Let $H^{\prime}=\sum_{\tau \in \mathcal{E}(H)} H_{\tau} \subseteq H$ and suppose that $\sum_{i=1}^{n} h_{\tau_{i}}=0$, where $h_{\tau_{i}} \in H_{\tau_{i}}, i=$ $1,2, \ldots, n$, and $\left\{\tau_{1}, \tau_{2}, \ldots, \tau_{n}\right\} \subseteq \mathcal{E}(H)$. By definition, there exists homomorphisms $\Psi_{i}: G \rightarrow H_{\tau_{i}}$ such that $\Psi_{i}\left(h_{\tau_{i}}\right)=h_{\tau_{i}}, i=1,2, \ldots, n$. If $\tau_{1}$, say, is minimal in $\left\{\tau_{1}, \tau_{2}, \ldots, \tau_{n}\right\}$, then

$$
0=\sum_{i=1}^{n} \Psi_{1}\left(h_{\tau_{i}}\right)=\Psi_{1}\left(h_{\tau_{1}}\right)=h_{\tau_{1}} .
$$

We conclude that $h_{\tau_{i}}=0, i=1,2, \ldots, n$, and therefore $H^{\prime}=\bigoplus_{\tau \in \mathcal{E}(H)} H_{\tau}$ is a completely decomposable group.

We now show that $H^{\prime}$ is strongly pure in $G$ (and therefore strongly pure in $H$ ). Let $h^{\prime}=\sum_{i=1}^{n} h_{\tau_{i}} \in H^{\prime}$, where $h_{\tau_{i}} \in H_{\tau_{i}}, i=1,2, \ldots, n$, and $\left\{\tau_{1}, \tau_{2}, \ldots, \tau_{n}\right\} \subseteq$ $\mathcal{E}(H)$. Then $\left\langle h_{\tau_{i}}\right\rangle_{*}$ is a summand of $G$ and is therefore a summand of some $\tau_{i^{-}}$ homogeneous summand of $G$. This implies that

$$
\sum_{i=1}^{n}\left\langle h_{\tau_{i}}\right\rangle_{*}=\bigoplus_{i=1}^{n}\left\langle h_{\tau_{i}}\right\rangle_{*}
$$

is a summand of $G$. Thus there exists a homomorphism $\Theta: G \rightarrow H^{\prime}$ such that $\boldsymbol{\theta}\left(h^{\prime}\right)=h^{\prime}$.

We shall now show that $H=H^{\prime}$, arguing as in the last paragraph of Theorem 98.3 in [2]. Let $h \in H$ be of type $\tau$; because the types in $T(H)$ satisfy the maximum condition we may assume that $\sum_{\tau^{\prime} \geq \tau} H\left(\tau^{\prime}\right) \subseteq H^{\prime}$. Since $H^{\prime}$ is pure in $G$, this implies that $\left\langle H^{*}(\tau)\right\rangle_{*} \subseteq H^{\prime}$. But $h \in H(\tau)=H_{\tau}^{\prime} \oplus\left\langle H^{*}(\tau)\right\rangle_{*} \subseteq H^{\prime}$ which therefore means that $H=H^{\prime}$ is completely decomposable.

The proof of Theorem 1 implies that if $H$ is a completely decomposable strongly pure subgroup of a completely decomposable group $G$, then the extractible typeset of $H$ is a subset of the extractible typeset of $G$. This is not necessarily true for pure completely decomposable subgroups of $G$. It also implies that if $\mathcal{E}$ is a subset of the extractible typeset of $G$ and $H_{\tau}$ is a pure subgroup of a maximal $\tau$-homogeneous summand of $G$ for every $\tau \in \mathcal{E}$, then $\sum_{\tau \in \mathcal{E}} H_{\tau}=\bigoplus_{\tau \in \mathcal{E}} H_{\tau}$ is a strongly pure subgroup of $G$. It is natural to ask whether all pure subgroups of completely decomposable groups arise in this way. Specifically, is every homogeneous 
strongly pure subgroup of $G$ a pure subgroup of some $\tau$-homogeneous summand of $G$ ? We shall give an answer to this question after we have considered strongly nice subgroups of separable torsion-free groups.

The following lemma will be crucial in the proof of our next theorem.

LEMMA 1. Let $G=\bigoplus_{i=1}^{n} G_{\tau_{i}} \oplus G^{\prime}$ be a completely decomposable group, where $0 \neq G_{\tau_{i}} \cong G\left(\tau_{i}\right) / G^{*}\left(\tau_{i}\right), i=1,2, \ldots, n$. Let $0 \neq g=\sum_{i=1}^{n} g_{i}+g^{\prime}$, where $g^{\prime} \in G^{\prime}$ and $0 \neq g_{i} \in G_{\tau_{i}}, i=1,2, \ldots, n$. Suppose that

(i) $\operatorname{type}_{G}\left(g^{\prime}\right) \geq \tau_{i}=\operatorname{type}_{G}\left(g_{i}\right), i=1,2, \ldots, n$, and

(ii) $\chi_{G}\left(g^{\prime}\right) \geq \bigcap_{i=1}^{n} \chi_{G}\left(g_{i}\right)$.

Then there exists a complement $\bigoplus_{i=1}^{n} G_{\tau_{i}}^{\prime}$ of $G^{\prime}$ in $G$ such that $G_{\tau_{i}}^{\prime} \cong G_{\tau_{i}}$ and $g=\sum_{i=1}^{n} g_{i}^{\prime}$, where $g_{i}^{\prime} \in G_{\tau_{i}}^{\prime}, i=1,2, \ldots, n$.

ProOF. For every $i \in\{1,2, \ldots, n\}$ define

$$
\mathbb{\Upsilon}_{i}=\left\{p(\text { prime }): h_{p}^{G}\left(g^{\prime}\right) \supsetneqq h_{p}^{G}\left(g_{i}\right)\right\} .
$$

Since type $\operatorname{s}_{G}\left(g^{\prime}\right) \geq \operatorname{type}_{G}\left(g_{i}\right)=\tau_{i}$, condition (i) implies that $\boldsymbol{\Phi}_{i}$ is finite for every $i \in\{1,2, \ldots, n\}$. Condition (ii) implies that $h_{p}^{G}\left(g^{\prime}\right) \geq \min \left\{h_{p}^{G}\left(g_{i}\right): i=1,2, \ldots, n\right\}$. We then have that $\bigcap_{i=1}^{n} \mathbb{\Upsilon}_{i}=\varnothing$. For every $i \in\{1,2, \ldots, n\}$ define

$$
r_{i}=\prod_{p \in \mathbb{I}_{i}} p^{\left(h_{p}^{G}\left(g_{i}\right)-h_{p}^{G}\left(g^{\prime}\right)\right)} .
$$

Since $\bigcap_{i=1}^{n} \mathbb{\Upsilon}_{i}=\varnothing$ the integers $r_{1}, r_{2}, \ldots, r_{n}$ are relatively prime. Thus there exists integers $s_{1}, s_{2}, \ldots, s_{n}$ such that $\sum_{i=1}^{n} r_{i} s_{i}=1$. This implies that $g^{\prime}=\sum_{i=1}^{n} r_{i} s_{i} g^{\prime}$. We now let $g_{i}^{\prime}=g_{i}+r_{i} s_{i} g^{\prime}$ for every $i \in\{1,2, \ldots, n\}$. Let $p$ be any prime.

(a) If $p \in \mathbb{I}_{i}$, we have

$$
h_{p}^{G}\left(r_{i} s_{i} g^{\prime}\right) \geq h_{p}^{G}\left(r_{i} g^{\prime}\right)=h_{p}^{G}\left(g^{\prime}\right)+h_{p}^{G}\left(g_{i}\right)-h_{p}^{G}\left(g^{\prime}\right)=h_{p}^{G}\left(g_{i}\right) .
$$

(b) If $p \notin \mathbb{q}_{i}$, we have

$$
h_{p}^{G}\left(r_{i} s_{i} g^{\prime}\right) \geq h_{p}^{G}\left(g^{\prime}\right) \geq h_{p}^{G}\left(g_{i}\right) .
$$

This implies that $\chi_{G}\left(g_{i}\right) \leq \chi_{G}\left(r_{i} s_{i} g^{\prime}\right)$ and therefore

$$
\chi_{G}\left(g_{i}^{\prime}\right)=\chi_{G}\left(g_{i}\right) \cap \chi_{G}\left(r_{i} s_{i} g^{\prime}\right)=\chi_{G}\left(g_{i}\right) .
$$

The proof of Theorem, 85.1 in [2] implies that there is an isomorphism $\Theta_{i}:\left\langle g_{i}\right\rangle_{*} \rightarrow$ $\left\langle g_{i}^{\prime}\right\rangle_{*}$ such that $\Theta_{i}\left(g_{i}\right)=g_{i}^{\prime}$. Since $G_{\tau_{i}}$ is a homogeneous completely decomposable group, $\left\langle g_{i}\right\rangle_{*}$ is a direct summand of $G_{\tau_{i}}$ with complement, say $X_{i}$. Let $\pi_{\tau_{i}}: G \rightarrow G_{\tau_{i}}$ $\left(=\left\langle g_{i}\right\rangle_{*} \oplus X_{i}\right)$ be a projection with $G^{\prime} \subseteq \operatorname{ker} \pi_{\tau_{i}}$ and define $G_{\tau_{i}}^{\prime}=\left\langle\left\{g_{i}^{\prime}, X_{i}\right\}\right\rangle_{*}$. If $h$ is an arbitrary element of $G$, then $h=g_{i}^{\prime \prime}+x_{i}+g_{i}^{\prime \prime \prime}$, where $g_{i}^{\prime \prime} \in\left\langle g_{i}\right\rangle_{*}, x_{i} \in X_{i}$ and $g_{i}^{\prime \prime \prime} \in \operatorname{ker} \pi_{\tau_{i}}$. There exists integers $m_{i}$ and $m_{i}^{\prime}$ such that

$$
m_{i}^{\prime} g_{i}^{\prime \prime}=m_{i} g_{i}=m_{i} \pi_{\tau_{i}}\left(g_{i}\right)=m_{i} \pi_{\tau_{i}}\left(g_{i}^{\prime}\right) .
$$

Let $h_{i}^{\prime}=\Theta_{i}\left(g_{i}^{\prime \prime}\right) \in\left\langle g_{i}^{\prime}\right\rangle_{*}$. This implies that

$$
m_{i}^{\prime} h_{i}^{\prime}=m_{i}^{\prime} \Theta_{i}\left(g_{i}^{\prime \prime}\right)=m_{i} \Theta_{i}\left(g_{i}\right)=m_{i} g_{i}^{\prime} .
$$

We then have $m_{i}^{\prime} g_{i}^{\prime \prime}=m_{i} \pi_{\tau_{i}}\left(g_{i}^{\prime}\right)=m_{i}^{\prime} \pi_{\tau_{i}}\left(h_{i}^{\prime}\right)$ and by torsion-freeness $g_{i}^{\prime \prime}=\pi_{\tau_{i}}\left(h_{i}^{\prime}\right)$. Hence $\pi_{\tau_{i}}(h)=\pi_{\tau_{i}}\left(g_{i}^{\prime \prime}+x_{i}\right)=\pi_{\tau_{i}}\left(h_{i}^{\prime}+x_{i}\right)$, which implies that $h-h_{i}^{\prime}-x_{i}=h_{i}^{\prime \prime} \in$ $\operatorname{ker} \pi_{\tau_{i}}$. Thus $h=h_{i}^{\prime}+x_{i}+h_{i}^{\prime \prime} \in G_{\tau_{i}}^{\prime}+\operatorname{ker} \pi_{\tau_{i}}$. We then have $G=G_{\tau_{i}}^{\prime}+\operatorname{ker} \pi_{\tau_{i}}$. We 
now let $h_{i} \in\left\langle\left\{g_{i}^{\prime}, X_{i}\right\}\right\rangle \cap \operatorname{ker} \pi_{\tau_{i}}$. Then $h_{i}=k g_{i}^{\prime}+x_{i}$ for some integer $k$ and $x_{i} \in X_{i}$ and

$$
0=\pi_{\tau_{i}}\left(h_{i}\right)=k \pi_{\tau_{i}}\left(g_{i}^{\prime}\right)+x_{i}=k g_{i}+x_{i} .
$$

Thus $k g_{i}=-x_{i} \in\left\langle g_{i}\right\rangle_{*} \cap X_{i}=\{0\}$ and by torsion-freeness $k=0$. Thus $h_{i}=0$ which implies that $G_{\tau_{i}}^{\prime} \cap \operatorname{ker} \pi_{\tau_{i}}=\{0\}$. We then have $G=G_{\tau_{i}}^{\prime} \oplus \operatorname{ker} \pi_{\tau_{i}}$ and $g_{i}^{\prime} \in G_{\tau_{i}}^{\prime} \cong G_{\tau_{i}}$. Since $G_{\tau_{i}}^{\prime}$ is a maximal $\tau_{i}$-homogeneous summand of $G$ for every $i \in\{1,2, \ldots, n\}$, then $\sum_{i=1}^{n} G_{\tau_{i}}^{\prime}=\bigoplus_{i=1}^{n} G_{\tau_{i}}^{\prime}$ is a summand of $G$ and its complement is $G^{\prime}$. Finally

$$
g=\sum_{i=1}^{n} g_{i}+g^{\prime}=\sum_{i=1}^{n}\left(g_{i}+r_{i} s_{i} g^{\prime}\right)=\sum_{i=1}^{n} g_{i}^{\prime}
$$

and the proof of the lemma is complete.

The following observation, deduced from the proof of the previous lemma, will be used repeatedly in the proof of the next theorem: let $G=\bigoplus_{\tau \in \mathcal{E}(G)} G_{\tau}$ be a homogeneous decomposition of a completely decomposable group $G$ and let $\pi_{\tau}: G \rightarrow$ $G_{\tau}$ be a projection. If $0 \neq g \in G$ satisfies $\chi_{G}(g)=\chi_{G}\left(\pi_{\tau}(g)\right)$ for some $\tau \in \mathcal{E}(G)$, then $g$ belongs to a maximal $\tau$-homogeneous summand of $G$.

We now prove

THEOREM 2. Let $H$ be a strongly pure subgroup of a completely decomposable group $G$. Then every element of $H$ is contained in a completely decomposable finite rank summand of $H$.

ProOF. Let $G=\bigoplus_{\tau \in \mathcal{E}(G)} G_{\tau}$ be a homogeneous decomposition of $G$ and let $\pi_{\tau}: G \rightarrow G_{\tau}$ be the projection with ker $\pi_{\tau}=\bigoplus_{\tau^{\prime} \neq \tau} G_{\tau^{\prime}}$, for every $\tau \in \mathcal{E}(G)$. For $0 \neq h \in H$ we let

$$
\mathcal{E}(h)=\left\{\tau \in \mathcal{E}(G): \pi_{\tau}(h) \neq 0\right\} .
$$

Our aim is to write $h$ in the form $h=\sum_{\tau \in \mathcal{E}(h)} h_{\tau}$, where, for every $\tau \in \mathcal{E}(h)$, $h_{\tau} \in H \cap G_{\tau}^{\prime}$ and $G_{\tau}^{\prime}$ is a maximal $\tau$-homogeneous summand of $G$.

We partition $\mathcal{E}(h)$ into subsets $\mathcal{M}_{k}, k=1,2, \ldots, d$, defined recursively as follows: $\mathcal{M}_{1}$ is the set of all minimal types in $\mathcal{E}(h)$ and, for $k=2,3, \ldots, d$, we say that $\tau \in \mathcal{E}(h)$ is in $\mathcal{M}_{k}$ if and only if there exists at least one $\tau^{\prime} \in \mathcal{M}_{k-1}$ satisfying $\tau^{\prime} \lessgtr \tau$, and, if $\tau^{\prime \prime}$ is any type in $\mathcal{E}(h)$ satisfying $\tau^{\prime} \lessgtr \tau^{\prime \prime} \leq \tau$, then either $\tau^{\prime \prime}=\tau$ or $\tau^{\prime \prime}=\tau^{\prime}$. We then have

$$
h=\sum_{k=1}^{d} \sum_{\tau \in \mathcal{M}_{k}} \pi_{\tau}(h) .
$$

Let $\Psi_{1}: G \rightarrow H$ be a homomorphism such that $\Psi_{1}(h)=h$. Then

$$
h=\sum_{k=1}^{d} \sum_{\tau \in \mathcal{M}_{k}} \Psi_{1}\left(\pi_{\tau}(h)\right) .
$$

Let $\tau \in \mathcal{M}_{1}$ and let $\tau^{\prime} \in \mathcal{E}(h)-\{\tau\}$. Then $\pi_{\tau} \Psi_{1} \pi_{\tau^{\prime}}(h)=0$ for, if $\pi_{\tau} \Psi_{1} \pi_{\tau^{\prime}}(h) \neq 0$ we would have $\tau^{\prime}=\operatorname{type}_{G}\left(\pi_{\tau^{\prime}}(h)\right) \leq \operatorname{type}_{G}\left(\pi_{\tau} \Psi_{1}\left(\pi_{\tau^{\prime}}(h)\right)\right)=\tau$ which contradicts the minimality of $\tau$ in $\mathcal{E}(h)$. Thus $\pi_{\tau}(h)=\pi_{\tau} \Psi_{1}\left(\pi_{\tau}(h)\right)$ for every $\tau \in \mathcal{M}_{1}$ and this implies that

$$
\chi_{G}\left(\pi_{\tau}(h)\right)=\chi_{G}\left(\pi_{\tau} \Psi_{1} \pi_{\tau}(h)\right) \geq \chi_{G}\left(\Psi_{1} \pi_{\tau}(h)\right) \geq \chi_{G}\left(\pi_{\tau}(h)\right) .
$$


Hence $\chi_{G}\left(\Psi_{1}\left(\pi_{\tau}(h)\right)\right)=\chi_{G}\left(\pi_{\tau} \Psi_{1} \pi_{\tau}(h)\right)$ and, by the observation after the proof of Lemma $1, \Psi_{1} \pi_{\tau}(h)=h_{\tau, 1}(\in H)$ is contained in a maximal $\tau$-homogeneous summand of $G$. We then have

$$
h=\sum_{\tau \in \mathcal{M}_{1}} h_{\tau, 1}+h_{2}^{\prime}, \quad \text { where } h_{2}^{\prime}=\sum_{\tau \in \mathcal{E}(h)-\mathcal{M}_{1}} \Psi_{1} \pi_{\tau}(h) \in \sum_{\tau \in \mathcal{M}_{2}} H(\tau) \subseteq \sum_{\tau \in \mathcal{M}_{2}} G(\tau) .
$$

Assume that, for a fixed $k \in\{2,3, \ldots, d\}$, we have expressed $h$ in the form

$$
h=\sum_{i=1}^{k-1} \sum_{\tau \in \mathcal{M}_{i}} h_{\tau, i}+h_{k}^{\prime}
$$

where $h_{\tau, i}(\in H)$ belongs to a maximal $\tau$-homogeneous summand of $G, \tau \in \mathcal{M}_{i}$ and $i=1,2, \ldots, k-1$, with $h_{k}^{\prime} \in \sum_{\tau \in \mathcal{M}_{k}} H(\tau) \subseteq \sum_{\tau \in \mathcal{M}_{k}} G(\tau)$. We now define

$$
\mathcal{E}\left(h_{k}^{\prime}\right)=\left\{\tau \in \mathcal{E}(G): \pi_{\tau}\left(h_{k}^{\prime}\right) \neq 0\right\}
$$

and observe that if $\mathcal{E}\left(h_{k}^{\prime}\right) \cap \mathcal{M}_{k} \neq \varnothing$, then it consists of some minimal types of $\mathcal{E}\left(h_{k}^{\prime}\right)$. In this case we let $\Psi_{k}^{\prime}: G \rightarrow H$ be a homomorphism such that $\Psi_{k}^{\prime}\left(h_{k}^{\prime}\right)=h_{k}^{\prime}$. Then $h_{k}^{\prime}=\sum_{\tau \in \mathcal{E}\left(h_{k}^{\prime}\right) \cap \mathcal{M}_{k}} \Psi_{k}^{\prime} \pi_{\tau}\left(h_{k}^{\prime}\right)+\sum_{\tau \in \mathcal{E}\left(h_{k}^{\prime}\right)-\mathcal{M}_{k}} \Psi_{k}^{\prime} \pi_{\tau}\left(h_{k}^{\prime}\right)$. By the first part of the proof of this theorem, $\Psi_{k}^{\prime}\left(\pi_{\tau}\left(h_{k}^{\prime}\right)\right)=h_{\tau, k}(\in H)$ belongs to a maximal $\tau$ homogeneous summand of $G$ for every $\tau \in \mathcal{E}\left(h_{k}^{\prime}\right) \cap \mathcal{M}_{k}$. Also, if $\tau \in \mathcal{E}\left(h_{k}^{\prime}\right)-\mathcal{M}_{k}$, then $\tau \supsetneqq \tau^{\prime}$ for some $\tau^{\prime} \in \mathcal{M}_{k}$ since $h_{k}^{\prime} \in \sum_{\tau \in \mathcal{M}_{k}} G(\tau)$. We then have

$$
h=\sum_{i=1}^{k} \sum_{\tau \in \mathcal{M}_{i}} h_{\tau, i}+h_{k}
$$

where

$$
h_{k}=\sum_{\tau \in \mathcal{E}\left(h_{k}^{\prime}\right)-\mathcal{M}_{k}} \Psi_{k}^{\prime} \pi_{\tau}\left(h_{k}^{\prime}\right) \in \sum_{\tau \in \mathcal{M}_{k}}\left\langle H^{*}(\tau)\right\rangle_{*} \subseteq \sum_{\tau \in \mathcal{M}_{k}} G^{*}(\tau) .
$$

We now let $\mathcal{E}\left(h_{k}\right)=\left\{\tau \in \mathcal{E}(G): \pi_{\tau}\left(h_{k}\right) \neq 0\right\}$ and define

$$
\begin{aligned}
& \mathcal{E}_{k}= \begin{cases}\left\{\tau \in \mathcal{E}\left(h_{k}\right): \tau \geq \tau^{\prime} \text { for some } \tau^{\prime} \in \mathcal{M}_{k+1}\right\}, & \text { if } 2 \leq k \leq d-1, \\
\varnothing & \text { if } k=d,\end{cases} \\
& \mathcal{E}_{k}^{\prime}=\mathcal{E}\left(h_{k}\right)-\mathcal{E}_{k} \text { if } 2 \leq k \leq d .
\end{aligned}
$$

Then $h_{k}=g_{k}+g_{k}^{\prime}$, where $g_{k}=\sum_{\tau \in \mathcal{E}_{k}} \pi_{\tau}\left(h_{k}\right)$ and $g_{k}^{\prime}=\sum_{\tau \in \mathcal{E}_{k}^{\prime}} \pi_{\tau}\left(h_{k}\right)$. We note that $\mathcal{E}(h) \cap \mathcal{E}_{k}^{\prime}=\varnothing, 2 \leq k \leq d$, and

$$
h=\sum_{i=1}^{k} \sum_{\tau \in \mathcal{M}_{i}} h_{\tau, i}+g_{k}+g_{k}^{\prime}
$$

For each $\tau^{\prime} \in \mathcal{E}_{k}^{\prime}$ define

$$
\mathcal{E}\left(\tau^{\prime}\right)=\left\{\tau \in \bigcup_{i=1}^{k} \mathcal{M}_{i}: \pi_{\tau^{\prime}}\left(h_{\tau, i}\right) \neq 0\right\} .
$$

We notice that if $\tau^{\prime} \in \mathcal{E}_{k}^{\prime}$ and $\pi_{\tau^{\prime}}\left(h_{\tau, i}\right) \neq 0$, where $\tau \in \bigcup_{i=1}^{k} \mathcal{M}_{i}$, then $\tau=$ type $_{G}\left(h_{\tau, i}\right) \supsetneqq \tau^{\prime}$ since $\tau \in \mathcal{E}(h)$ and $\mathcal{E}(h) \cap \mathcal{E}_{k}^{\prime}=\varnothing$. We now define a relation $\sim$ on $\mathcal{E}_{k}^{\prime}$ by requiring that $\tau_{1}^{\prime} \sim \tau_{2}^{\prime}$ if and only if $\mathcal{E}\left(\tau_{1}^{\prime}\right)=\mathcal{E}\left(\tau_{2}^{\prime}\right)$ for any two types $\tau_{1}, \tau_{2}$ in 
$\mathcal{E}_{k}^{\prime}$. This is obviously an equivalence relation on $\mathcal{E}_{k}^{\prime}$ and we let $\left\{\mathcal{E}_{k, 1}^{\prime}, \ldots, \mathcal{E}_{k, n_{k}}^{\prime}\right\}$ be the set of all equivalence classes.

We can associate with each equivalence class $\mathcal{E}_{k, m}^{\prime}$, where $m=1,2, \ldots, n_{k}$, a uniquely determined subset, say $\mathcal{M}\left(\mathcal{E}_{k, m}^{\prime}\right)$, of $\bigcup_{i=1}^{k} \mathcal{M}_{i}$ defined by

$$
\mathcal{M}\left(\mathcal{E}_{k, m}^{\prime}\right)=\left\{\tau \in \bigcup_{i=1}^{k} \mathcal{M}_{i}: \pi_{\tau^{\prime}}\left(h_{\tau, i}\right) \neq 0 \text { for every } \tau^{\prime} \in \mathcal{E}_{k, m}^{\prime}\right\} .
$$

Then $\pi_{\tau^{\prime}}\left(h_{\tau^{\prime \prime}, i}\right)=0$ for every $\tau^{\prime} \in \mathcal{E}_{k, m}^{\prime}$ and every $\tau^{\prime \prime} \in\left(\bigcup_{i=1}^{k} \mathcal{M}_{i}\right)-\mathcal{M}\left(\mathcal{E}_{k, m}^{\prime}\right)$. Put

$$
g_{k}^{\prime}=\sum_{\tau \in \mathcal{E}_{k}^{\prime}} \pi_{\tau}\left(g_{k}^{\prime}\right)=\sum_{m=1}^{n_{k}} g_{k, m}^{\prime}
$$

where

$$
g_{k, m}^{\prime}=\sum_{\tau \in \mathcal{E}_{k, m}^{\prime}} \pi_{\tau}\left(g_{k}^{\prime}\right), \quad m=1,2, \ldots, n_{k} .
$$

Then, for every $\tau^{\prime} \in \mathcal{E}_{k, m}^{\prime}$, we have

$$
0=\pi_{\tau^{\prime}}(h)=\pi_{\tau^{\prime}}\left(\sum_{\tau \in \mathcal{M}\left(\mathcal{E}_{k, m}^{\prime}\right)} h_{\tau, i}\right)+\pi_{\tau^{\prime}}\left(g_{k, m}^{\prime}\right)
$$

which implies that

$$
\begin{aligned}
\chi_{G}\left(\pi_{\tau^{\prime}}\left(g_{k, m}^{\prime}\right)\right) & =\chi_{G}\left(\pi_{\tau^{\prime}}\left(\sum_{\tau \in \mathcal{M}\left(\mathcal{E}_{k, m}^{\prime}\right)} h_{\tau, i}\right)\right) \\
& \geq \chi_{G}\left(\sum_{\tau \in \mathcal{M}\left(\mathcal{E}_{k, m}^{\prime}\right)} h_{\tau, i}\right)=\bigcap_{\tau \in \mathcal{M}\left(\mathcal{E}_{k, m}^{\prime}\right)} \chi_{G}\left(h_{\tau, i}\right) .
\end{aligned}
$$

Thus

$$
\chi_{G}\left(g_{k, m}^{\prime}\right)=\bigcap_{\tau^{\prime} \in \mathcal{E}_{k, m}^{\prime}} \chi_{G}\left(\pi_{\tau^{\prime}}\left(g_{k, m}^{\prime}\right)\right) \geq \bigcap_{\tau \in \mathcal{M}\left(\mathcal{E}_{k, m}^{\prime}\right)} \chi_{G}\left(h_{\tau, i}\right)
$$

and

$$
\operatorname{type}_{G}\left(g_{k, m}^{\prime}\right)=\bigcap_{\tau^{\prime} \in \mathcal{E}_{k, m}^{\prime}} \tau^{\prime} \geq \tau=\operatorname{type}_{G}\left(h_{\tau, i}\right)
$$

for every $\tau \in \mathcal{M}\left(\mathcal{E}_{k, m}^{\prime}\right)$.

Let $\Psi_{k}: G \rightarrow H$ be a homomorphism such that $\Psi_{k}\left(h_{k}\right)=h_{k}$. Then

$$
\begin{aligned}
h & =\sum_{i=1}^{k} \sum_{\tau \in \mathcal{M}_{i}} h_{\tau, i}+h_{k}=\sum_{i=1}^{k} \sum_{\tau \in \mathcal{M}_{i}} h_{\tau, i}+\Psi_{k}\left(h_{k}\right) \\
& =\sum_{i=1}^{k} \sum_{\tau \in \mathcal{M}_{i}} h_{\tau, i}+\Psi_{k}\left(g_{k}\right)+\Psi_{k}\left(g_{k}^{\prime}\right) \\
& =\sum_{i=1}^{k} \sum_{\tau \in \mathcal{M}_{i}} h_{\tau, i}+\Psi_{k}\left(g_{k}\right)+\sum_{m=1}^{n_{k}} \Psi_{k}\left(g_{k, m}^{\prime}\right)
\end{aligned}
$$


Consider, for example, $\sum_{\tau \in \mathcal{M}\left(\mathcal{E}_{k, 1}^{\prime}\right)} h_{\tau, i}+\Psi_{k}\left(g_{k, 1}^{\prime}\right)$ and observe that

(i) type $\tan _{G}\left(\Psi_{k}\left(g_{k, 1}^{\prime}\right)\right) \geq \operatorname{type}_{G}\left(g_{k, 1}^{\prime}\right) \geq \tau=\operatorname{type}_{G}\left(h_{\tau, i}\right)$ for every $\tau \in \mathcal{M}\left(\mathcal{E}_{k, 1}^{\prime}\right)$ and

(ii) $\chi_{G}\left(\Psi_{k}\left(g_{k, 1}^{\prime}\right)\right) \geq \chi_{G}\left(g_{k, 1}^{\prime}\right) \geq \bigcap_{\tau \in \mathcal{M}\left(\mathcal{E}_{k, 1}^{\prime}\right)} \chi_{G}\left(h_{\tau, i}\right)$.

Then, by Lemma 1 ,

$$
\sum_{\tau \in \mathcal{M}\left(\mathcal{E}_{k, 1}^{\prime}\right)} h_{\tau, i}+\Psi_{k}\left(g_{k, 1}^{\prime}\right)=\sum_{\tau \in \mathcal{M}\left(\mathcal{E}_{k, 1}^{\prime}\right)} h_{\tau, i}^{\prime},
$$

where $h_{\tau, i}^{\prime}$ belongs to a $\tau$-homogeneous summand of $G$. Also, from the proof of Lemma $1, \chi_{G}\left(h_{\tau, i}\right)=\chi_{G}\left(h_{\tau, i}^{\prime}\right)$ for every $\tau \in \mathcal{M}\left(\mathcal{E}_{k, 1}^{\prime}\right)$ and $\left\{h_{\tau, i}^{\prime}: \tau \in \mathcal{M}\left(\mathcal{E}_{k, 1}^{\prime}\right)\right\} \subseteq H$. Repeat this process for the elements

$$
\sum_{\tau \in \mathcal{M}\left(\mathcal{E}_{k, m}^{\prime}\right)} h_{\tau, i}+\Psi_{k}\left(g_{k, m}^{\prime}\right), \quad 2 \leq m \leq n_{k} .
$$

(N.B. For the sake of simplicity of notation, we put $h_{\tau, i}^{\prime}=h_{\tau, i}$ for $\tau \in \mathcal{M}\left(\mathcal{E}_{k, 1}^{\prime}\right)$ and adopt this convention of using the symbol $h_{\tau, i}$ for all the components of $h$ belonging to maximal $\tau$-homogeneous summands of $G$ and obtained by the process described above for $m=1$.)

We will then be able to express $h$ in the form

$$
h=\sum_{i=1}^{k} \sum_{\tau \in \mathcal{M}_{i}} h_{\tau, i}+h_{k+1}^{\prime}
$$

where $h_{\tau, i}(\in H)$ belongs to a maximal $\tau$-homogeneous summand of $G, \tau \in \mathcal{M}_{i}, i=$ $1,2, \ldots, k$, and $h_{k+1}^{\prime}=\Psi_{k}\left(g_{k}\right) \in H \cap \sum_{\tau \in \mathcal{M}_{k+1}} G(\tau)$. This expression of $h$ is similar to expression (A). We can repeat the process for the integers $k+1, k+2, \ldots, d$. We note that, by definition, $\mathcal{E}_{d}=\varnothing$ and therefore $g_{d}=\sum_{\tau \in \mathcal{E}_{d}} \pi_{\tau}\left(h_{k}\right)=0$. Thus $h_{d+1}^{\prime}=\Psi_{d}\left(g_{d}\right)=0$ which implies that

$$
h=\sum_{i=1}^{d} \sum_{\tau \in \mathcal{M}_{i}} h_{\tau, i}
$$

Since $h_{\tau, i}(\in H)$ belongs to a maximal $\tau$-homogeneous summand of $G$ for every $\tau \in \mathcal{E}(h)$, it is easy to see that $\sum_{\tau \in \mathcal{E}(h)}\left\langle h_{\tau, i}\right\rangle_{*}=\bigoplus_{\tau \in \mathcal{E}(h)}\left\langle h_{\tau, i}\right\rangle_{*}$ is a finite rank summand of $G$. Thus $\bigoplus_{\tau \in \mathcal{E}(h)}\left\langle h_{\tau, i}\right\rangle_{*}$ is a completely decomposable finite rank summand of $H$ containing $h$ and the proof of the theorem is complete.

The classical theorem of Kulikov on completely decomposable groups assets that countable direct summands of completely decomposable groups are completely decomposable. With the help of Theorem 2, we can extend this theorem to strongly pure subgroups of completely decomposable groups. The idea of the proof of the next theorem comes from the proof of Theorem 2 in Kolettis's paper Homogeneously decomposable modules (Studies on Abelian Groups, Springer, Berlin, 1968, pp. 223-238).

THEOREM 3. Countable strongly pure subgroups of completely decomposable groups are completely decomposable.

PROOF. Let $G$ be a completely decomposable group and $H$ a countable strongly pure subgroup of $G$ and let $\left\{h_{1}, h_{2}, \ldots, h_{n}\right\}$ be a finite subset of $H$. Then, by 
Theorem 2 above, $H=H_{1} \oplus H_{1}^{\prime}$, where $H_{1}$ is a finite rank completely decomposable group containing $h_{1}$. Let $h_{2}^{\prime}$ be the projection of $h_{2}$ on $H_{1}^{\prime}$. Since $H_{1}^{\prime}$ is also strongly pure in $G$, Theorem 2 implies that $H_{1}^{\prime}=H_{2} \oplus H_{2}^{\prime}$, where $H_{2}$ is a finite rank completely decomposable group containing $h_{2}^{\prime}$. Proceeding in this manner we obtain a finite rank completely decomposable summand $H_{k+1}$ of $H_{k}^{\prime}$ for $2 \leq k \leq n$, which contains the projection $h_{k+1}^{\prime}$ of $h_{k+1}$ on $H_{k}^{\prime}$ where $H=\bigoplus_{i=1}^{k} H_{i} \oplus H_{k}^{\prime}$ with $\bigoplus_{i=1}^{k} H_{i}$ a finite rank completely decomposable group and $H_{k}^{\prime}$ is obviously strongly pure in $G$. This implies that $H$ is separable since $\bigoplus_{i=1}^{n} H_{i}$ will be a finite rank completely decomposable summand of $H$ containing $\left\{h_{1}, h_{2}, \ldots, h_{n}\right\}$. Since $H$ is countable, $H$ is necessarily completely decomposable.

The proof of Theorem 3 implies that strongly pure subgroups of completely decomposable groups are separable. We recall that pure subgroups of homogeneous separable groups are strongly pure. Thus strongly pure subgroups of homogeneous separable groups are separable by Corollary 87.3 in [2]. The classical theorem of Fuchs on separable torsion-free groups asserts that direct summands of separable torsion-free groups are separable. All these results are immediate corollaries of the following theorem.

THEOREM 4. Strongly pure subgroups of separable torsion-free groups are separable.

ProOF. Let $G$ be a separable torsion-free group, $H$ strongly pure in $G$ and let $\left\{h_{1}, h_{2}, \ldots, h_{n}\right\}$ be a finite subset of $H$. There exists a finite rank completely decomposable summand, $G_{1}$, of $G$ such that $\left\{h_{1}, h_{2}, \ldots, h_{n}\right\} \subseteq G_{1}$. By definition, there exists a homomorphism $\Psi_{1}: G \rightarrow H$ such that $\Psi_{1}(h)=h$ for every $h \in H \cap G_{1}$. Therefore $H \cap G_{1} \subseteq G_{1} \subseteq\left\langle\Psi_{1}\left(G_{1}\right), G_{1}\right\rangle_{*}$ and the latter is a finite rank pure subgroup of $G$. Thus, there exists a finite rank completely decomposable summand, $G_{2}$, of $G$ such that $\left\langle\Psi_{1}\left(G_{1}\right), G_{1}\right\rangle_{*} \subseteq G_{2}$ and there exists a homomorphism $\Psi_{2}: G \rightarrow$ $H$ such that $\Psi_{2}(h)=h$ for every $h \in H \cap G_{2}$.

Let $m$ be an integer satisfying $m>1$ and assume that we have obtained an ascending chain

$$
G_{1} \subseteq G_{2} \subseteq \cdots \subseteq G_{k} \subseteq \cdots \subseteq G_{m}
$$

of finite rank completely decomposable summands of $G$ and homomorphism $\Psi_{k}: G \rightarrow H$ such that $\Psi_{k}(h)=h$ for every $h \in H \cap G_{k}, 1 \leq k \leq m$. Then $\left\langle\sum_{k=1}^{m} \Psi_{k}\left(G_{m}\right), G_{m}\right\rangle_{*}$ is a finite rank pure subgroup of $G$ containing $G_{m}$, which is contained in a finite rank completely decomposable summand $G_{m+1}$ of $G$. Let $\Psi_{m+1}: G \rightarrow H$ be a homomorphism such that $\Psi_{m+1}(h)=h$ for every $h \in H \cap$ $G_{m+1}$. Repeat the process $\omega$ times. We then obtain an ascending sequence

$$
G_{1} \subseteq G_{2} \subseteq \cdots \subseteq G_{n} \subseteq \cdots, \quad n<\omega,
$$

of finite rank summands of $G$ and homomorphisms $\Psi_{n}: G \rightarrow H$ such that $\Psi_{n}(h)=h$ for every $h \in H \cap G_{n}, n<\omega$. Let $G^{\prime}=\bigcup_{n<\omega} G_{n}$. Since $G^{\prime}$ is the union of an ascending sequence of strongly pure subgroups of $G$ and strong purity is an inductive property, $G^{\prime}$ is strongly pure in $G$. It is easy to see that $G^{\prime}$ is a countable completely decomposable group. We shall now prove that $H^{\prime}=H \cap G^{\prime}$ is strongly pure in $G^{\prime}$.

Let $0 \neq h^{\prime} \in H^{\prime}$. Then there exists an integer, $k$ say, such that $h^{\prime} \in H \cap G_{k}$ and, by definition, $\Psi_{k}\left(h^{\prime}\right)=h^{\prime}$. Let $\Psi_{k}^{\prime}$ be the restriction of $\Psi_{k}$ to $G^{\prime}$ and let $g^{\prime}$ be 
an arbitrary element of $G^{\prime}$. Then there exists an integer, say $r$, such that $g^{\prime} \in G_{r}$. Then

(a) if $r \leq k$, we have

$$
\Psi_{k}^{\prime}\left(g^{\prime}\right)=\Psi_{k}\left(g^{\prime}\right) \in H \cap\left\langle\sum_{i=1}^{k} \Psi_{i}\left(G_{k}\right), G_{k}\right\rangle_{*} \subseteq H \cap G_{k+1} \subseteq H^{\prime},
$$

(b) if $r \supsetneqq k$, we have

$$
\Psi_{k}^{\prime}\left(g^{\prime}\right)=\Psi_{k}\left(g^{\prime}\right) \in H \cap\left\langle\sum_{i=1}^{r} \Psi_{i}\left(G_{r}\right), G_{r}\right\rangle_{*} \subseteq H \cap G_{r+1} \subseteq H^{\prime} .
$$

From (a) and (b) we conclude that $\Psi_{k}^{\prime} \in \operatorname{Hom}\left(G^{\prime}, H^{\prime}\right)$ and $\Psi_{k}^{\prime}\left(h^{\prime}\right)=h^{\prime}$.

Thus $H^{\prime}$ is a countable strongly pure subgroup of $G^{\prime}$. Since $G^{\prime}$ is completely decomposable, Theorem 3 asserts that $H^{\prime}$ is also completely decomposable. Also, since strong purity is transitive, $H^{\prime}$ is strongly pure in $G$. Obviously $\left\{h_{1}, h_{2}, \ldots, h_{n}\right\} \subseteq$ $H^{\prime}$ and therefore there is a finite rank summand $H_{1}$ of $H^{\prime}$ containing $\left\{h_{1}, h_{2}, \ldots\right.$, $\left.h_{n}\right\}$. We observe that $H_{1}$ is strongly pure in $G$. Thus $H_{1}$ is a finite rank completely decomposable summand of $H$ containing $\left\{h_{1}, h_{2}, \ldots, h_{n}\right\}$ and therefore $H$ is separable.

Let $G$ be a group, $H$ a subgroup of $G$ and $\beta: G \rightarrow G / H$ the natural epimorphism. Then $H$ is said to be strongly nice in $G$ if, for every $g \in G$, there exists a homomorphism $\Psi: G / H \rightarrow G$ such that $\beta \Psi \beta(g)=\beta(g)$. This concept was introduced by $\mathrm{K}$. M. Rangaswamy and one of the results he obtained is the following:

A strongly nice subgroup of a separable group is strongly pure.

We are going to use this result to find an example of a homogeneous strongly pure subgroup of a completely decomposable group $G$ which is not a subgroup of any maximal homogeneous summand of $G$.

Let $\tau_{1}$ and $\tau_{2}$ be any two types with $\tau_{1} \neq(\infty, \infty, \ldots, \infty)$ and $\tau_{2} \supsetneqq \tau_{1}$. Theorem 5 in [5] states that there exists an $\aleph_{1}$-separable group $A$ such that the typeset of $A$ is $\left\{\tau_{1}, \tau_{2}\right\}$ and $A$ is not a direct sum of homogeneous groups. There exists a balanced exact sequence

$$
0 \rightarrow H \rightarrow G \rightarrow A \rightarrow 0
$$

where $G$ is a completely decomposable group with $\mathcal{E}(G)=\left\{\tau_{1}, \tau_{2}\right\}$. It can be shown that $H$ is strongly nice in $G$. Thus $H$ is strongly pure in $G$ and by Theorem $1, H$ is completely decomposable and the extractible typeset of $H$ is a subset of $\left\{\tau_{1}, \tau_{2}\right\}$. Let $H=H_{\tau_{1}} \oplus H_{\tau_{2}}$, where $H_{\tau_{i}}$ is a homogeneous completely decomposable group of type $\tau_{i}, i=1,2$, and assume that there is a homogeneous decomposition $G=G_{\tau_{1}} \oplus G_{\tau_{2}}$ of $G$ such that $H_{\tau_{i}} \subseteq G_{\tau_{i}}, i=1,2$. This will imply that $A \cong G / H \cong$ $\left(G_{\tau_{1}} / H_{\tau_{1}}\right) \oplus\left(G_{\tau_{2}} / H_{\tau_{2}}\right)$. It is easy to show that $G_{\tau_{i}} / H_{\tau_{i}}$ is a homogeneous group of type $\tau_{i}, i=1,2$. Thus $A$ is a direct sum of homogeneous groups, a contradiction. Hence $H_{\tau_{2}}$ is a homogeneous strongly pure subgroup of $G$ which is not contained in any maximal homogeneous summand of $G$.

We have not been able to determine whether or not strongly pure (or even strongly nice) subgroups of completely decomposable groups are completely decomposable. In view of Theorems 1 and 3 a natural step in this direction is to determine whether or not strongly pure subgroups of completely decomposable groups are direct sums of countable groups (cf. Kaplansky's Theorem on direct 
summands of direct sums of countable groups). In [3] Hill derived a necessary and sufficient condition for an isotype subgroup of a direct sum of countable $p$-groups ( $p$ a prime) to be itself a direct sum of countable groups. It has been shown in [4] that isotype subgroups of totally projective $p$-groups are strongly pure. Therefore, Example 1 in [3] implies that strongly pure subgroups of direct sums of countable groups are not necessarily direct sums of countable groups. It seems likely that there is a strongly pure subgroup of a completely decomposable group which is not completely decomposable.

Let $G$ be a torsion-free group which is the union of an ascending sequence of strongly pure subgroups $G_{n}, n<\omega$. If each $G_{n}$ is completely decomposable, is $G$ also completely decomposable? It is easy to show that if $G$ is countable, then $G$ will be completely decomposable. Hill has proved an analogous theorem which states that if $G$ is a $p$-group which is the set-theoretic union of a countable collection of isotype totally projective subgroups $G_{n}$, then $G$ is totally projective.

ACKNOWLEDGEMENT. This paper forms part of the author's D.Phil. thesis submitted at Oxford University in 1982. I would like to thank my supervisor Dr. A. L. S. Corner for his assistance in its preparation.

\section{REFERENCES}

1. H. Bowman and K. M. Rangaswamy, On special balanced subgroups of torsion-free separable groups, Abelian Group Theory (Proceedings, Oberwolfach 1981), Lecture Notes in Math., vol. 874, Springer-Verlag, Berlin and New York, 1981, pp. 32-40.

2. L. Fuchs, Infinite abelian groups, Vol. 2, Academic Press, New York, 1973.

3. P. Hill, Isotype subgroups of totally projective groups, Abelian Group Theory (Proceedings, Oberwolfach 1981), Lecture Notes in Math., vol. 874, Springer-Verlag, Berlin and New York, 1981, pp. 305-321.

4. S. Janakiraman and K. M. Rangaswamy, Strongly pure subgroups of abelian groups (Proc. Miniconf. Theory of Groups, Canberra 1977), Lecture Notes in Math., vol. 574, Springer-Verlag, Berlin and New York, 1977, pp. 57-64.

5. A. H. Mekler, $\aleph_{1}$-separable groups of mixed type, Abelian Group Theory (Proceedings, Oberwolfach 1981), Lecture Notes in Math., vol. 874, Springer-Verlag, Berlin and New York, 1981, pp. 114-126.

MATHEMATICS DePARTMENT, NATIONAL UNIVERSity OF LESOThO, P.O. ROMA 180, LESOTHO, SOUTHERN AFRICA 